\title{
A New Design in Parabolic Trough Using Heat Retention
}

\author{
R. Maruthi Prasad*, Surendra Prasad, Sanatana Ravi, Narsimha Kota. \\ Rural Development Society, Prestige Rai Towers, Flat No.404, B-Block, Punjagutta, Hyderabad, Telangana, \\ and India.
}

Abstract: The paper gives the summary of new design of parabolic trough using heat retention method. It gives a brief idea of on heat retention and describes about the components and operation of parabolic trough. This is an updating of parabolic trough collector system. The advantages collectors are also discussed.

\section{Introduction}

At present a parabolic trough is a type of solar thermal energy collector. It is constructed as a long parabolic mirror with a Dewar Tube running its length at the focal point. Sun Light is reflected by the mirror and concentrated on the Dewar Tube.

This tracking method works correctly at the spring and falls equinoxes with errors in the focusing light at other times during the year. This method also reaches minimum solar noon, due to this parabolic troughs are generally designed with a lower solar concentration ratio in order to increase alignment. Cosine losses also involve in the reduction of overall efficiency of the collector. In case of focusing collector out of beam and diffused solar radiation components, only beam component is collected because diffuse component cannot be reflected back on to the absorber and is thus lost.

These problems can be overcome to large extent and also thermal ficiency of the collector can be increased by using the design below which employs the concept of Total Internal Reflection.

\section{Methodology}

At present parabolic trough is employing reflection property. Besides these properties the collector Introduced in this paper uses the concept of Heat Retention.

\section{Design Explanation}

The design consists of three main parts, they are I) High transfer Plastic sheet, 2) glass slab and 3) Silver polymer sheet. The arrangements of the above components are shown in fig2. The detailed explanation of each part is given below.

\section{1) High Transfer plastic fiber:}

These are made of specialized designed fiber with certain cross section of internal side which is placed over the parabolic trough collector which allows maximum light to pass through. It also helps to reflect back the diffused component coming from the dewar tubes.

\section{Glass Slab:-}

The cross sectioned area of High transfer plastic fiber sheet side is covered by a glass layer of required refractive index. The most commonly used optical glasses refractive index Thus by employing a glass layer on the ranges from 1.52 to 1.72 . To obtain cross section side of the plastic fiber reflection of the diffused light the light we can avoid the loss of reflected light ray must be diverged at an angle component. greater than 41.8 degrees as shown in fig.

$$
\begin{aligned}
& \sin \theta_{r}=\frac{n_{1}}{n_{2}} \sin \theta_{i} \quad \text { From Snells Law } \\
& \theta_{c}=\theta_{i}=\arcsin \left(\frac{n_{2}}{n_{1}}\right),
\end{aligned}
$$

$\mathrm{Qc}=\arcsin (1.00 / 1.50)-41.8$

The angle obtained above is the critical angle; if a light ray incident above the critical angle it will be reflected back. Glass layer of low reflectivity and high transmissivity are desired to keep the amount of reflected and absorbed radiation low. The glass layer and the plastic fiber should have same refractive index so that they behave as one medium. Instead of the glass layer we can keep plastic layer in order to reduce weight and cost of 
panel. A stagnant air gap interposes high impedance to convective heat flow between the absorber and the ambient air. The losses, both of radiation and convection, can be reduced to low values.

Thus by employing a glass layer on the cross section side of the plastic fiber we can avoid the loss of reflected light component.

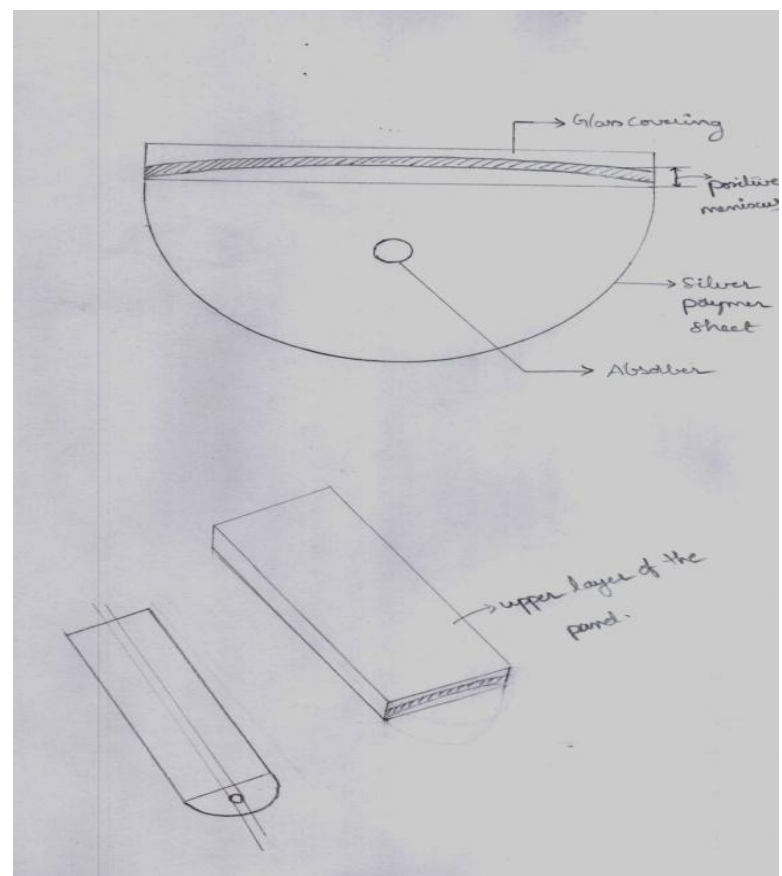

Fig.2 Arrangement of parts to give an outlook of a solar panel

\section{Silver Polymer Sheet:-}

The Silver Polymer sheet arrangement is shown in fig. the purpose of using the Sll'ver Sheet is to focus the reflected light from the absorber back on to the absorber.

Usually mirrors are used which are parabolic and are of a single piece in addition V-type parabolic trough exist which are made from two mirrors and placed at an angle towards each other. This parabolic trough can be replaced by a silver polymer sheet that has the same performance as heavy glass mirror but at a much lower cost and much lower weight. The glossy film uses several layers of polymer with an inner layer of pure silver. It is also much easier to deploy and install.

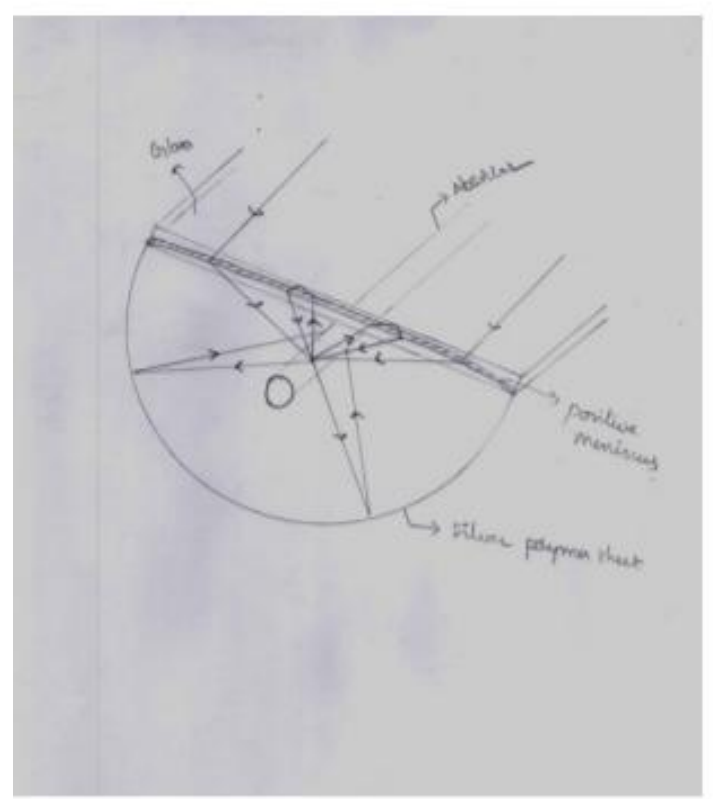

Fig.3. Capturing of both beam and diffused light 


\section{Advantages Of This Design}

1. As the collector is completely closed the stagnant air gap interposes high impedance to convective heat flow between the absorber and the ambient air

2. The losses of both radiation and convection can be reduced.

3. Both beam and iffused component of solar radiation is collected.

4. By using the silver olymer sheet the weight of the panel is reduced, so that we can employ the rotating system for better tracking.

5. The absorber can attain high temperature compared to parabolic troughs.

6. As both beam and diffused radiation are captured the efficiency of collector is increased there by collector to grid efficiency also increases.

7. We can use plastic sheet of reflective property instead of glass to obtain total internal Reflection there by the cost and weight of collector is reduced.

8. The collector can give satisfying results even in bad weather conditions because heat lost to the environment is less.

9. Performance of the collector can be increased by replacing the stagnant air gap with vacuum.

10. As the collector is completely closed and vacuum is employed by inside it, the maintenance of the panel like protection from oxidation, antifreeze coating clearing leaves are not required

\section{Conclusion}

In this paper a new design has been proposed to increase the efficiency of a parabolic trough collector. Though it may appear complex, but the use of this panel yields high temperature steam and also reduce the maintenance of panels in case of dirt, weather etc. as well as heat losses to the atmosphere is reduced. So it might serve as better alternative to parabolic trough collectors.

\section{References}

[1]. A Text Book on power plant engineering by A.K.Rajput

[2]. A Text Book on Solar Energy Utilization by G.D Rao

[3]. A Text book on Solar Energy Fundamentals and Applications by H.P Garg and J. Prakash

[4]. Award winning Solar collectors will cut production cost www.energyboom.cor

[5]. Wind and Solar power system by patel. 